\title{
A Critical Examination of the Polysymptomatic Distress Scale Construct as a Symptom Severity Questionnaire
}
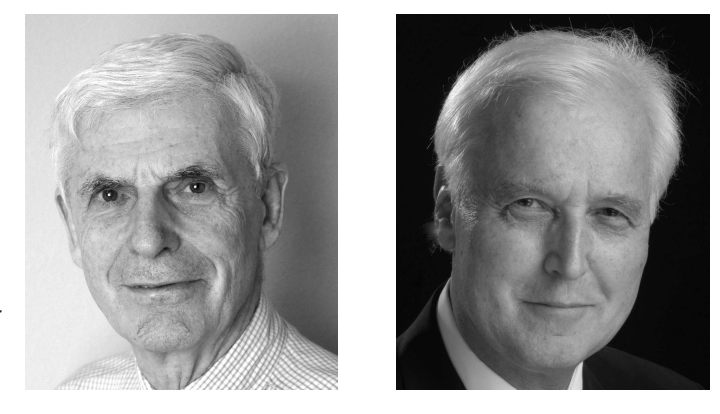

A confusion develops between the use of scores to measure severity, and the same scores to establish diagnosis. Hugh A. Smythe

J Rheumatol 2011;38:975-8

The assessment of disease severity is an essential undertaking related to morbidity and mortality. Ideally such assessment involves the use of objective markers, such as the level of hemoglobin in anemia or distribution of the number of erosions in rheumatoid arthritis. In many diseases within the sphere of rheumatology, objective markers have yet to be discovered. This situation has led to a profusion of questionnaires aimed at measuring disease severity. Such questionnaires need to be carefully designed to address the disorder under scrutiny and comprehensively validated to ensure their scientific reliability.

The main symptom in most rheumatological disorders is pain. There are currently no generally available objective measures of pain, and its assessment invariably relies upon questionnaires, such as the Brief Pain Inventory ${ }^{1}$, or scales such as the visual analog scale ${ }^{2,3}$. Further, the evaluation of pain is complicated by its multidimensionality; most patients with chronic pain are fatigued, commonly depressed, often functionally impaired, and existentially distressed. The development of questionnaires has become a specialty in its own right, with its own arcane vocabulary, statistical complexities, and even its own journals. Achieving the right balance of generality and specificity without loss of content or efficiency is a challenging undertaking.

In this issue of The Journal, Wolfe and colleagues present the Polysymptomatic Distress Scale (PSD) as a useful general severity measure and advocate its ease of interpretation by assigning 5 severity categories (none, mild, moderate, severe, and very severe $)^{4}$. The PSD is derived from Dr. Wolfe's 2010 diagnostic criteria for fibromyalgia $(\mathrm{FM})^{5}$. It combines 2 scales used for diagnosis: the Widespread Pain Index (WPI;
0-19) and the Symptom Severity Scale (SSS; 0-12) for a combined PSD total of $0-31^{6}$. The WPI consists of 19 nonarticular regions assessed for their presence/absence of pain. The SSS contains 6 symptoms: fatigue, sleep, cognition, headache, abdominal pain/cramping, and depression. "Polysymptomatic Distress" is the newest term for the scales previously named FM Symptom and "Fibromyalgianess", although there has been no change in content. The authors commend the PSD as a multipurpose instrument: (a) a measure of FM severity, (b) a "universal quantity" for assessing symptom severity for all disorders, (c) an "approximate diagnosis" of FM, and (d) an instrument for clinical and research purposes. To quote:

We suggest that the distribution of PSD represents an aspect of the human condition, i.e., some patients report more pain and distress and some less, and PSD can be seen as a broad continuous distribution.... We also note that using the continuous PSD scale rather than classifying patients into FM or widespread pain groups makes it easier to understand the relationship between variables and the degree of the patient's problem, and patients on both sides of the FM or widespread pain dichotomy are often more similar than different ${ }^{7}$.

The development of a scientifically valid, widely accepted questionnaire, presents many difficult choices that influence its ultimate acceptance and validity. There are 3 issues that are worth considering when using the PSD: the method for determining pain severity, the methods used in the derivation of clinically relevant symptoms, and the disproportionate influence of pain locations relative to symptoms in the final score.

Pain locations as a measure of pain severity. The PSD relies on the 19-point WPI component as its primary assessment of pain. However, the WPI is inherently underrepresentative of pain regions; in its development only nonarticular regions

See Polysymptomatic distress categories, page 1494

Personal non-commercial use only. The Journal of Rheumatology Copyright @ 2015 . All rights reserved. 
were selected, and articular ones eliminated based on statistical criteria with the goal of FM classification ${ }^{8}$. While the selection of axial and near axial regions seems reasonable for FM classification purposes, it poses a problem when the primary source of pain, such as joint pain, is not represented in an instrument that claims to assess severity in rheumatic disorders in general. An equally important problem is the WPI reliance on the number of pain sites as a measure of pain severity, with the assumption that more pain sites equate with more pain intensity. By definition, therefore, disorders that have more pain sites will necessarily have more pain than disorders with fewer pain sites. On the other hand, for disorders with fewer pain sites, there is little room in the WPI pain count for a disorder with high pain intensity, such as gout or migraine.

A third problem is the underrepresentation of pain qualities in the PSD. This is apparent when the WPI is compared to other scales. For example, the Revised Fibromyalgia Impact Questionnaire (FIQR)/Symptom Impact Questionnaire (SIQR) includes stiffness and tenderness to touch as well as pain intensity ${ }^{9}$. In the 2010 FM diagnostic study "the WPI and muscle tenderness were the most important variables in the classification of cases and non-cases"5. In the 2013 FM diagnostic study, tenderness to touch was an important differentiating symptom ${ }^{10}$. However, the 2010 FM construct "evolved" by eliminating "muscle tenderness" and "muscle pain" symptoms as important in defining FM. Muscle tenderness was present in $79 \%$ of FM-positive patients; nevertheless, it was excluded from the final criteria set because the WPI alone satisfied American College of Rheumatology (ACR) classification using statistical criteria ${ }^{5}$. The rationale for this is not entirely clear because "tender muscles" (1-0) had a slightly better correlation with FM diagnosis than did the 19-point WPI ( 0.507 vs 0.492$)^{6}$. Interestingly "muscle pain" and "muscle tenderness" were not included in the SSS, but 3 symptoms (headaches, abdominal pain/cramping, and depression) were included even though they had lower variable importance and correlations with ACR 1990-defined diagnosis of FM. Notably, the binary item "tender muscles," which differentiates FM from non-FM patients, was displaced by the "continuous" WPI spectrum variable. These substitutions lack face validity and are not consonant with findings of the OMERACT (Outcome Measures in Rheumatology) studies ${ }^{11}$. The transformation of "tender muscles" from a global FM symptom into the summation of discrete continuous pain location scores (i.e., WPI) underlies an unstated conceptual change: a potentially unique symptom

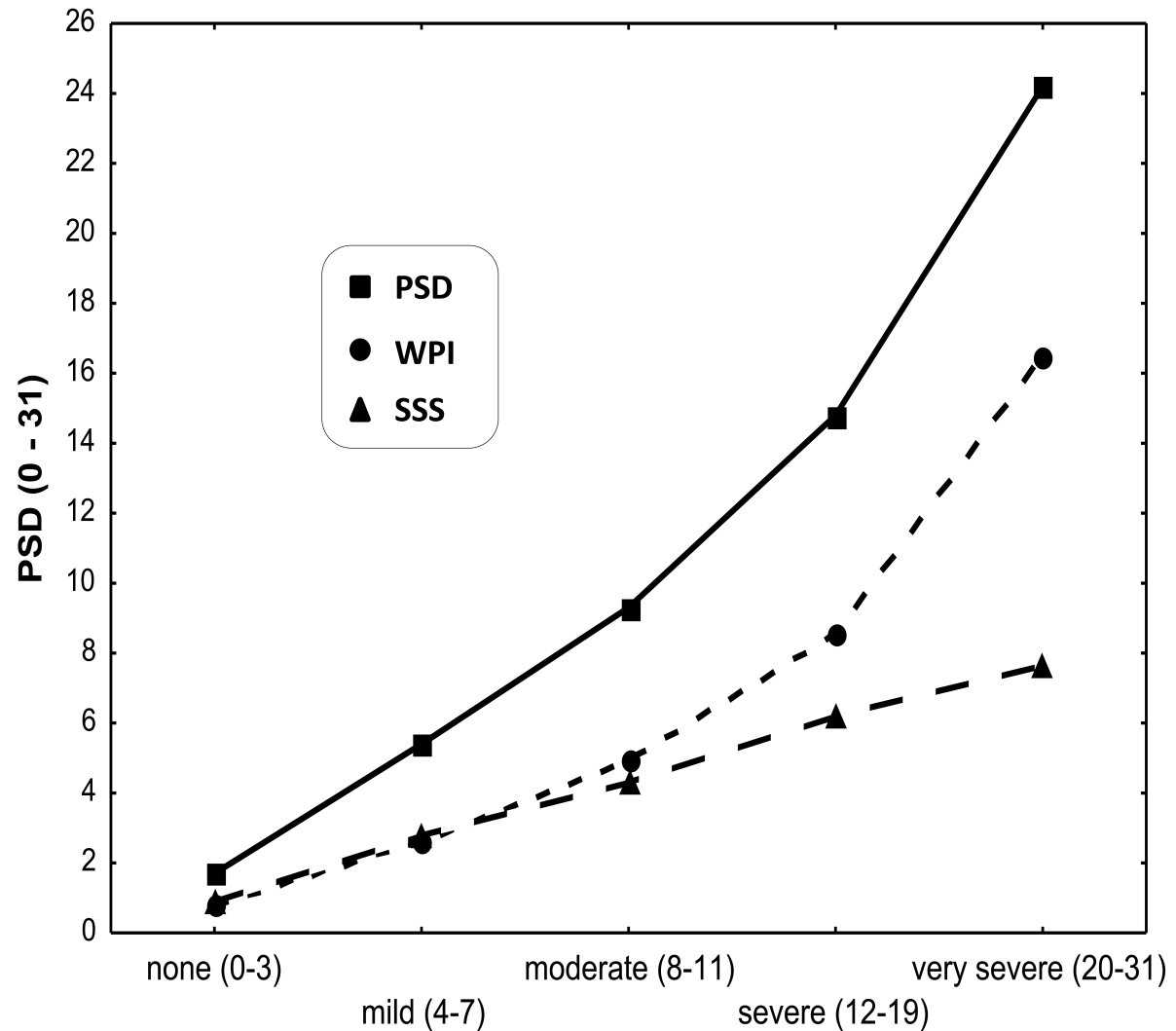

\section{Effect Sizes}

\begin{tabular}{llllll}
\hline WPI & --- & 1.57 & 1.45 & $1.43(1.52)$ & $2.72(3.18)$ \\
SSS & --- & 1.58 & 0.94 & $0.94(1.61)$ & $0.65(0.53)$
\end{tabular}

Figure 1. Mean values for PSD, WPI, and SSS by severity category*. The contribution of the SSS and WPI to the PSD score are about the same for the "none," "mild," and "moderate" categories*. However, as severity increases, the WPI component exerts a disproportionately greater influence on the PSD score, and the severity categories cease to have a uniform distribution. The effect sizes (comparing a category mean with its preceding category mean) shows a diminishing effect of the SSS, accompanied by an increase for the WPI from low to high severity categories. Thus the PSD has different meanings across different severity categories, making the categories difficult to interpret.

*Values are taken from Tables 1 and 2 and the supplementary table in the accompanying article (Wolfe, et al, Reference 4). Effect sizes in parentheses are for the subset of patients in "severe" and "very severe" categories who are 2010 FM-positive (i.e., 3-6 WPI and $\geq 9$ SSS or $\geq 7$ WPI and $\geq 5$ SSS). PSD: Polysymptomatic Distress Scale; WPI: Widespread Pain Index; SSS: Symptom Severity Scale; FM: fibromyalgia.

Personal non-commercial use only. The Journal of Rheumatology Copyright @ 2015 . All rights reserved. 
differentiating FM was thus transformed into a continuous quantitative spectrum based on statistical criteria rather than clinical criteria.

SSS as a measure of non-pain symptoms. The SSS is composed of 3 symptoms (fatigue, sleep, and cognition) assessed over the past month on a $0-3$ scale, and 3 symptoms (headache, abdomen pain/cramps, and depression) assessed over the past 6 months for their presence or absence $(0 / 1)$, for a total score of 12 . As with the WPI, these 6 symptoms were selected from a larger set of symptoms, based on empirical-statistical criteria that were ranked according to their importance in classifying patients with FM from those without it. This larger set did not include symptoms that the extant clinical and research literature had found to be pertinent to the assessment of severity, as for example, stiffness $^{12,13}$, balance ${ }^{14}$, tenderness to touch ${ }^{15}$, environmental sensitivity ${ }^{16,17}$, and physical functioning ${ }^{18,19}$. Physical functioning was omitted: "because our scales were designed primarily for aiding in diagnosis"6, i.e., as with the WPI, items were selected for classification, not severity.

Problems with the PSD in assessing disease severity. The aim of the accompanying article by Wolfe and colleagues was to provide severity categories to "translate potentially unfamiliar PSD scores" for clinicians and investigators. However, the greater theoretical weighting given to the WPI (range 0-19) than to the SSS (range 0-12) becomes even more problematic when the actual component scores are applied to the severity categories, making this aim difficult to achieve. In Figure 1, we plot the PSD, WPI, and SSS means from the Wolfe, et al article, Tables 1 and $2^{4}$. It is seen that the contribution of WPI and SSS to the PSD is uneven across severity categories, showing small linear increase of SSS and WPI at lower categories of equal magnitude, and an accelerating contribution of the WPI relative to the SSS of differing magnitude with increasing severity. The irregular effect sizes comparing SSS and WPI means (Figure 1) will be particularly problematic for clinicians and researchers attempting to interpret symptom and pain site changes across time and severity categories. These differences are due to the wider ranges in higher severity categories ( 8 and 12 vs 4 ) compounded by a disproportionate influence of WPI/SSS weighting. Overall the PSD is a narrowly defined severity construct despite many correlates [global severity, SF-36 (Medical Outcomes Study Short Form-36 questionnaire) physical component, generalized anxiety disorder, etc.]. These "covariates" are not surrogates that can effectively replace the direct assessment of an inclusive symptom set, based on comprehensively validated findings such as those found in the OMERACT studies ${ }^{20}$.

The PSD is unique among severity questionnaires by being an offshoot of a disease classification questionnaire. This has resulted in 3 issues that need consideration when using the scale: The use of pain locations to measure pain severity, the omission of several symptoms related to disease severity, and the disproportionate influence of pain locations relative to symptoms in the final score. We would suggest that our own scale, the SIQR, a disease-neutral version of the $\mathrm{FIQR}^{21,22}$, could be considered as an alternative.

\author{
RONALD FRIEND, $\mathrm{PhD}$, \\ Professor of Psychology, \\ Stony Brook University, \\ Stony Brook, New York; and \\ Adjunct Professor of Nursing, \\ Oregon Health and Sciences University, \\ Portland, Oregon, USA;
}

ROBERT M. BENNETT, MD,

Professor of Medicine and Nursing,

Oregon Health and Science University,

Portland, Oregon, USA.

Address correspondence to R. Friend, Oregon Health and Sciences University, 2347 NW Overton St., Portland, Oregon 97210, USA

E-mail: ronald.friend@stonybrook.edu

This work is supported by the Fibromyalgia Information Foundation.

\section{REFERENCES}

1. Cleeland CS, Ryan KM. Pain assessment: global use of the Brief Pain Inventory. Ann Acad Med Singapore 1994;23:129-38.

2. Crawford BK, Piault EC, Lai C, Bennett RM. Assessing fibromyalgia-related fatigue: content validity and psychometric performance of the Fatigue Visual Analog Scale in adult patients with fibromyalgia. Clin Exp Rheumatol 2011;29 Suppl 69:S34-43.

3. Raspe H, Cellarius J, Mau W, Wasmus A, von Gierke S. [Guidelines for social medicine assessment and evaluation of primary fibromyalgia]. [Article in German] Gesundheitswesen 1994; 56:596-8.

4. Wolfe F, Walitt BT, Rasker JJ, Katz RS, Häuser W. The use of polysymptomatic distress categories in the evaluation of fibromyalgia (FM) and FM severity. J Rheumatol 2015;42:1494-501.

5. Wolfe F, Clauw DJ, Fitzcharles MA, Goldenberg DL, Katz RS, Mease P, et al. The American College of Rheumatology preliminary diagnostic criteria for fibromyalgia and measurement of symptom severity. Arthritis Care Res 2010;62:600-10.

6. Wolfe F, Clauw DJ, Fitzcharles MA, Goldenberg DL, Hauser W, Katz RS, et al. Fibromyalgia Criteria and Severity Scales for clinical and epidemiological studies: a modification of the ACR Preliminary Diagnostic Criteria for Fibromyalgia. J Rheumatol 2011;38:1113-22.

7. Wolfe F, Michaud K, Busch RE, Katz RS, Rasker JJ, Shahouri SH, et al. Polysymptomatic distress in patients with rheumatoid arthritis: understanding disproportionate response and its spectrum. Arthritis Care Res 2014;66:1465-71.

8. Wolfe F. Pain extent and diagnosis: development and validation of the regional pain scale in 12,799 patients with rheumatic disease. J Rheumatol 2003;30:369-78.

9. Bennett RM, Friend R, Jones KD, Ward R, Han BK, Ross RL. The Revised Fibromyalgia Impact Questionnaire (FIQR): validation and psychometric properties. Arthritis Res Ther 2009;11:R120.

10. Bennett RM, Friend R, Marcus D, Bernstein C, Han BK, Yachoui R, et al. Criteria for the diagnosis of fibromyalgia: validation of the modified 2010 preliminary American College of Rheumatology criteria and the development of alternative criteria. Arthritis Care Res 2014;66:1364-73.

11. Mease PJ, Clauw DJ, Christensen R, Crofford LJ, Gendreau RM, Martin SA, et al. Toward development of a fibromyalgia responder index and disease activity score: OMERACT module update. J Rheumatol 2011;38:1487-95.

Personal non-commercial use only. The Journal of Rheumatology Copyright $\odot$ (2015. All rights reserved 
12. Bennett R, Russell IJ, Choy E, Spaeth M, Mease P, Kajdasz D, et al. Evaluation of patient-rated stiffness associated with fibromyalgia: a post-hoc analysis of 4 pooled, randomized clinical trials of duloxetine. Clin Ther 2012;34:824-37.

13. Bellamy N, Sothern RB, Campbell J. Aspects of diurnal rhythmicity in pain, stiffness, and fatigue in patients with fibromyalgia. J Rheumatol 2004;31:379-89.

14. Jones KD, Horak FB, Winters-Stone K, Irvine JM, Bennett RM. Fibromyalgia is associated with impaired balance and falls. J Clin Rheumatol 2009;15:16-21.

15. Amris K, Waehrens EE, Jespersen A, Stockmarr A, Bennett R, Bliddal $\mathrm{H}$, et al. The relationship between mechanical hyperalgesia assessed by manual tender point examination and disease severity in patients with chronic widespread pain: A cross-sectional study. Int J Rheumatol 2014;2014:417596.

16. Wilbarger JL, Cook DB. Multisensory hypersensitivity in women with fibromyalgia: implications for well being and intervention. Arch Phys Med Rehabil 2011;92:653-6.

17. Carrillo-de-la-Pena MT, Vallet M, Perez MI, Gomez-Perretta C. Intensity dependence of auditory-evoked cortical potentials in fibromyalgia patients: a test of the generalized hypervigilance hypothesis. J Pain 2006;7:480-7.
18. Arnold LM, Zlateva G, Sadosky A, Emir B, Whalen E. Correlations between fibromyalgia symptom and function domains and patient global impression of change: a pooled analysis of three randomized, placebo-controlled trials of pregabalin. Pain Med 2011;12:260-7.

19. Assumpcao A, Sauer JF, Mango PC, Pascual MA. Physical function interfering with pain and symptoms in fibromyalgia patients. Clin Exp Rheumatol 2010;28 Suppl 63:S57-63.

20. Boers M, Kirwan JR, Gossec L, Conaghan PG, D'Agostino MA, Bingham CO III, et al. How to choose core outcome measurement sets for clinical trials: OMERACT 11 approves filter 2.0. J Rheumatol 2014;41:1025-30.

21. Friend R, Bennett RM. Distinguishing fibromyalgia from rheumatoid arthritis and systemic lupus in clinical questionnaires: an analysis of the revised Fibromyalgia Impact Questionnaire (FIQR) and its variant, the Symptom Impact Questionnaire (SIQR), along with pain locations. Arthritis Res Ther 2011;13:R58.

22. Overview of the FIQR and SIQR. [Internet. Accessed June 8, 2015.] Available from: http://fiqrinfo.ipage.com/

J Rheumatol 2015;42:1364-7; doi:10.3899/jrheum.150611 